


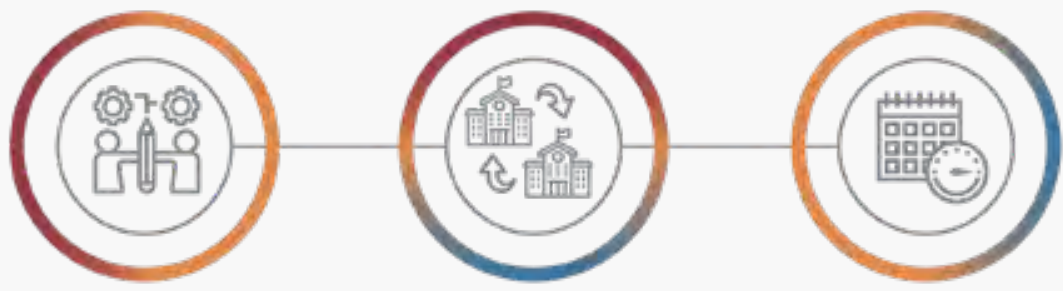

\section{CAPÍTULO III \\ ANÁLISIS PROSPECTIVO DEL SECTOR MANUFACTURERO CASO EMPRESA RODILLOS KHAN}

Claudia Patricia Delgadillo Hernández Jhonatan Adrian Rodriguez Sierra Allynson Venegas Camargo 


\section{DISEÑO DEL ESTUDIO DE CASO}

Rodillos Khan es una empresa que se dedica a la producción y comercialización de rodillos. En este documento se evidencia la aplicación de una herramienta de prospectiva. Esta herramienta nos permite visualizar la empresa objeto de estudio en varios escenarios posibles por medio de un análisis que tiene diferentes etapas.

El análisis comienza por los factores críticos de éxito. Dichos factores fueron revisados, actualizados y reestructurados para ser aplicados. Fueron escogidos 10 de un total de factores, por medio de un ranking y de su magnitud de impacto. Posteriormente se evaluaron en la matriz de impactos cruzados, que consiste en asignar una calificación a cada factor dependiendo si este influye o depende en los demás. Esto arrojó una lista de variables que hacen parte de la matriz de hipótesis, en la cual se plantearon tres resultados potenciales por variable o factor crítico de éxito con su respectivo porcentaje de probabilidad para que este sea alcanzable. Finalmente, gracias a la matriz de escenarios se obtuvieron 15 escenarios posibles con su respectiva hipótesis asociada a cada uno de los factores críticos de éxito. De allí se seleccionaron 3 escenarios por el grupo de trabajo para proponer una estrategia con su respectivo plan táctico para su cumplimiento.

\section{PALABRAS CLAVE}

Internacionalización, estrategia, escenarios, prospectiva.

\section{ANTECEDENTES}

Rodillos Khan es una empresa colombiana dedicada a la fabricación de rodillos para pintar y todos los implementos de plástico necesarios para esta labor. Pertenece al sector manufacturero y se encuentra ubicada en la ciudad de Bogotá. Cuenta con presencia a nivel nacional y exporta a Perú y Ecuador. Además, distribuye sus productos a través de las ferreterías y tiendas de pintura. 
Inicialmente se identificaron algunos factores críticos durante el diagnóstico otorgado:

Para el proceso de producción se cuenta con 12 máquinas que funcionan con energía eléctrica. La organización no cuenta con una planta eléctrica en caso de suspensión de la energía eléctrica, lo cual afecta directamente la producción. Tiene un único proveedor de felpa realizada especialmente para Rodillos Khan, es decir, si el proveedor suspende sus actividades la organización no puede solucionar fácilmente.

La empresa tiene grandes existencias (stocks). Pero sus productos no tienen una correcta rotación afectando su flujo de caja. La infraestructura de la empresa es irregular debido a que la estructura tiene techos de madera, significando un riesgo para sus operarios y personal que visite la fábrica.

En el mes de mayo de 2018 la producción real del sector manufacturero y de plásticos creció $7.1 \%$ con relación al mismo mes de 2017. Durante dicho mes las ventas reales crecieron $4.0 \%$ y el empleo manufacturero disminuyó $1.5 \%$. Como refleja el Ministerio de Comercio, Industria y Turismo: "Para el periodo enero a mayo de 2018, la producción real del sector industrial se incrementó en $2.7 \%$, las ventas reales crecieron $3.8 \%$, por otra parte, el empleo manufacturero se redujo -1.8\%." (Mincit, 2019).

El sector de la construcción aumentó en 5,1\% en el primer trimestre, debido a la mayor ejecución de obras en viviendas, edificios, carreteras, calles, caminos y otras construcciones del sector privado y público, según informó el Instituto Nacional de Estadística e Informática (INEI) (Redacción EC, 2018). Este importante aumento de la construcción en Perú significó un crecimiento sustancial para las exportaciones de los productos de Rodillos Khan a este país debido a que, al incrementar las construcciones, crece la demanda de pinturas e implementos para su aplicación.

Rodillos Khan cuenta con una felpa de excelente calidad fabricada exclusivamente para esta empresa. Ello genera preferencia de la marca entre los compradores por su durabilidad en el momento de la aplicación de la pintura. El buen manejo de la logística de distribución de esta empresa le permite ofrecer tiempos de entrega 
más rápidos, distribuyendo la mercancía al cliente en un tiempo máximo de un día para la ciudad de Bogotá y dos días para el resto del territorio nacional; marcando un nivel de preferencia al momento de compra para sus clientes por su agilidad y compromiso. El servicio posventa comprende la solución oportuna de reclamos e inquietudes, los servicios de mantenimiento, el cumplimiento de las garantías o el otorgamiento de nuevas facilidades, la calidez en la atención y las encuestas de satisfacción.

\section{Misión}

"Somos fabricantes de rodillos para pintar, que buscan por medio de la calidad satisfacer las necesidades del pintor profesional o del hogar, en las principales ciudades de Colombia, Ecuador y Perú".

\section{Visión}

"Industrias Khan será reconocida en el largo plazo como una empresa líder en el mercado nacional e internacional por ofrecer una completa gama de productos que atiendan las necesidades del pintor profesional o del hogar, con productos de alta calidad, durabilidad y a un precio justo, con una amplia red de distribución nacional e internacional".

\section{UNIDAD DE ANÁLISIS}

Rodillos Khan es una empresa dedicada a la fabricación de rodillos para pintar y productos plásticos para la misma actividad. En su línea de productos cuenta con 7 tipos diferentes de rodillos, con diferencias en el tipo de felpa y el diseño. Adicionalmente, cuenta con repuestos para rodillos, veteadores que permiten realizar una técnica de pintura efecto madera, cubetas para aplicar la pintura de manera uniforme en el rodillo, espátulas y brochas.

Actualmente distribuye sus productos a nivel nacional y hacia algunos países como Perú y Ecuador, donde obtiene beneficios arancelarios debido a la participación de Colombia en la CAN (Comunidad Andina de Naciones); en donde también se encuentra Perú y Ecuador. 
Su objetivo corporativo es la expansión del mercado en el exterior. Inicialmente la empresa desea llegar a países como Brasil, Honduras, Costa Rica y Guatemala; con el método de entrada de exportación directa.

Se realizó una búsqueda de las posibilidades de exportar a estos países. Con base en los resultados se determinó que la mejor opción es exportar a Bolivia. Esto debido a que, al igual que Perú y Ecuador, esto se debe a que este país hace parte de la CAN al igual que Perú y Ecuador lo cual representa beneficios arancelarios.

Según el Ministerio de Economía y Finanzas Públicas de Bolivia (2017), en este país se pronosticó un crecimiento que para el 2018 representó un 4,7\%; a comparación del crecimiento del PIB de Colombia que fue de 2,7 \% (Becerra, 2019). Para el sector de construcción el crecimiento en Bolivia en 2018 fue de 7\%, siendo el sector con mayor crecimiento en este país.

\section{MÉTODOS PARA INFORMACIÓN}

Para conocer el estado actual de la compañía y a donde se quiere llegar se deben responder las siguientes preguntas:

\section{¿Dónde estamos?}

Rodillos Khan es una empresa dedica a la venta de rodillos e implementos para pintar, actualmente tiene ventas en Colombia y en el exterior; donde su principal cliente es Ecuador y exporta a Perú en menores cantidades.

Cuenta con aproximadamente 1500 clientes entre ferreterías y tiendas de pintura a nivel nacional. En Ecuador y Perú, la compañía presento un crecimiento del $2.69 \%$ en el año 2017.

\section{¿Cómo estamos?}

Una de sus mayores debilidades es su portafolio de productos poco variado en comparación a su competencia, quedando en desventaja ya que la competencia abarca mayor mercado. Adicionalmente, sus instalaciones no son seguras para las personas que trabajan allí debido a la construcción del lugar. 
Por otro lado, podemos observar una de sus mayores fortalezas en la calidad del producto ya que cuenta con un proveedor de felpa exclusivo para la fabricación de los rodillos y con un servicio posventa que garantiza la calidad del producto, una respuesta rápida y oportuna que deja a sus clientes satisfechos.

Actualmente el objetivo de la empresa es aumentar la participación en el mercado nacional y tener mayor presencia en el exterior.

Para el año 2017, la empresa tuvo unos ingresos de 6.480 millones.

\section{¿Dónde queremos ir?}

Rodillos Khan quiere llegar a ser una empresa reconocida por la calidad de sus productos. Aspira a ser líder en el mercado nacional e internacional contando con una amplia red de distribución para colocar sus productos en almacenes como ferreterías, almacenes de cadena y grandes superficies. La empresa quiere llegar a nuevos mercados como Brasil, Honduras, Costa Rica y Guatemala.

\section{¿Hacia dónde debemos ir?}

Rodillos Khan primero debe enfocarse en tener una mayor participación a nivel nacional, comenzando con una diversificación concéntrica creando alianzas para vender y distribuir productos al cliente final de manera directa. De esta manera, se incrementa la rentabilidad y se permanece al nivel de la competencia contando con una variedad de productos relacionados con la construcción. Se aprovecharán canales de distribución en el exterior, así como el crecimiento del sector construcción en países como Panamá (9\%) y Bolivia (7\%).

\section{¿Hacia dónde podemos ir?}

Después del crecimiento del año 2017, la empresa no ha presentado ningún cambio. Se plantea realizar un plan de mercadeo, evaluar nuevos distribuidores a nivel nacional y aumentar su portafolio de productos para tener una mayor participación.

\section{¿Hacia dónde vamos?}

Mayor participación en el mercado con un producto de calidad, con mayor diversificación en el portafolio con el fin de ofrecer 
variedad de productos al cliente final, así como aumento de la participación (principalmente en Colombia).

Adicionalmente, la empresa puede aprovechar las oportunidades de crecimiento del sector de construcción en el exterior; y de esta manera, convertirse en una de las empresas líderes en venta de rodillos y accesorios para pintura complementando su portafolio con productos relacionados con la construcción.

También se proyecta como una de las empresas líderes en exportación, principalmente en la CAN.

\section{MÉTODOS DE ANÁLISIS}

A continuación, se presenta paso a paso el método de análisis empleado por medio de la prospectiva para la empresa:

\section{Identificación de factores críticos de éxito}

Identificación de factores los cuales se clasifican como oportunidades, fortalezas, amenazas y debilidades que la empresa tiene actualmente o que afectan a la empresa. Se hace una descripción de cada uno y se asigna un indicador que permita medir el cumplimiento de los factores.

El segundo paso es dar un orden para hallar los primeros 10 factores según la magnitud del impacto. La magnitud del impacto es calificada de 1 a 3. De acuerdo con dicha calificación se posicionan en un ranking. A continuación, los 10 factores clasificados según ámbito tecnológico, económico, interno o social. 


\section{Tabla 1. Tendencias y descripción}

\begin{tabular}{|c|c|}
\hline NOMBRE VARIABLE & DESCRIPCIÓN \\
\hline $\begin{array}{c}\text { El precio de la energía en } \\
\text { Colombia aumentó en } 12,55 \% \\
\text { para el } 2019 .\end{array}$ & $\begin{array}{l}\text { Variación del IPC anual en Bogotá: } \\
12,55 \% \text { en el precio de la energía. Con } \\
\text { respecto a la capital del país, según } \\
\text { el DANE, la variación de las tarifas de } \\
\text { la energía para el mes de diciembre } \\
\text { estuvo por encima de los arrenda- } \\
\text { mientos, alimentación y pensiones } \\
\text { (Redacción Portafolio, 2019). }\end{array}$ \\
\hline $\begin{array}{c}\text { Productos sustitutos (pistolas de } \\
\text { pintura de turbina y compresor, } \\
\text { rodillos recargables). }\end{array}$ & $\begin{array}{l}\text { La pistola de pintura funciona } \\
\text { con base en una corriente aire } \\
\text { comprimido que atomiza el líquido } \\
\text { contenido en un depósito para tal } \\
\text { fin. El líquido (que también puede } \\
\text { ser pintura, aceite, entre otros) } \\
\text { permite ser aplicado sobre cual- } \\
\text { quier superficie de modo uniforme, } \\
\text { logrando un buen acabado y } \\
\text { terminado. De esta manera, se } \\
\text { logra pintar grandes superficies } \\
\text { con rapidez y contar con acabados } \\
\text { más homogéneos. Existen pistolas } \\
\text { eléctricas de turbina y compresor. } \\
\text { Su uso requiere de espacios } \\
\text { ventilados. También se necesita un } \\
\text { disolvente adecuado para rebajar } \\
\text { la pintura y accionar la pistola a } 20 \\
\text { cm. de la superficie a pintar (Leroy } \\
\text { Merlin, 2019). }\end{array}$ \\
\hline $\begin{array}{l}\text { Calidad del producto, mano } \\
\text { de obra calificada }\end{array}$ & $\begin{array}{l}\text { Por medio de la mano de obra } \\
\text { calificada se garantiza un producto } \\
\text { de calidad que cumpla con las } \\
\text { expectativas del cliente. También } \\
\text { se cuenta con un proveedor que } \\
\text { ofrece una felpa de mayor calidad } \\
\text { exclusivamente para Rodillos Khan. }\end{array}$ \\
\hline
\end{tabular}




\begin{tabular}{|c|l|}
\hline & El sector de la construcción se \\
& encuentra en constante creci- \\
& miento. Para 2019, se estimó que en \\
Colombia crecerá entre el 3,1\% y el & 4,1\% según la Cámara Colombiana \\
de la Construcción. Similar ten- \\
dencia se pronosticó para el resto \\
de países integrantes de la CAN \\
(Flórez, 2019).
\end{tabular}


Portafolio poco variado

En comparación con su competencia el portafolio de Rodillos Khan es menos variado.

Tabla 2. Factores e indicadores

\begin{tabular}{|c|c|c|c|c|}
\hline FACTOR & $\begin{array}{l}\text { NOMBRE } \\
\text { VARIABLE }\end{array}$ & INDICADOR & DOFA & $\begin{array}{c}\text { MAGNITUD } \\
\text { DEL IMPACTO }\end{array}$ \\
\hline Económico & $\begin{array}{l}\text { El precio de } \\
\text { la energía } \\
\text { en Colombia } \\
\text { aumentó en } \\
\text { un } 12,55 \% \\
\text { para el } 2019 \text {. }\end{array}$ & $\begin{array}{l}\text { Porcentaje de } \\
\text { aumento en } \\
\text { el precio de la } \\
\text { energía. }\end{array}$ & A & 3 \\
\hline Tecnológico & $\begin{array}{l}\text { Productos } \\
\text { sustitutos } \\
\text { (pistolas de } \\
\text { pintura de } \\
\text { turbina y de } \\
\text { compresor, } \\
\text { rodillos } \\
\text { recargables). }\end{array}$ & $\begin{array}{l}\text { Cantidad de } \\
\text { productos } \\
\text { sustitutos } \\
\text { incluidos en el } \\
\text { portafolio). }\end{array}$ & A & 3 \\
\hline Interno & $\begin{array}{l}\text { Calidad del } \\
\text { producto, } \\
\text { mano de obra } \\
\text { calificada. }\end{array}$ & $\begin{array}{l}\text { Porcentaje de } \\
\text { los productos } \\
\text { que no cum- } \\
\text { plen con los } \\
\text { estándares de } \\
\text { calidad. }\end{array}$ & & \\
\hline $\begin{array}{l}\text { Número de } \\
\text { productos } \\
\text { defectuosos } \\
\text { / Número } \\
\text { total de } \\
\text { productos * } \\
100\end{array}$ & $F$ & 3 & & \\
\hline Económico & $\begin{array}{l}\text { Sector en } \\
\text { crecimiento. }\end{array}$ & $\begin{array}{l}\text { Porcentaje de } \\
\text { crecimiento del } \\
\text { sector. }\end{array}$ & $\mathrm{O}$ & 3 \\
\hline
\end{tabular}




\begin{tabular}{|c|c|c|c|c|}
\hline Interno & $\begin{array}{l}\text { Canales de } \\
\text { distribución } \\
\text { dentro y fuera } \\
\text { del país. }\end{array}$ & $\begin{array}{l}\text { Promedio de } \\
\text { tiempos de } \\
\text { entrega. }\end{array}$ & $\mathrm{F}$ & 2 \\
\hline Tecnológico & $\begin{array}{l}\text { Rodillo } \\
\text { eléctrico. }\end{array}$ & $\begin{array}{l}\text { Cantidad de } \\
\text { productos } \\
\text { sustitutos } \\
\text { incluidos en el } \\
\text { portafolio. }\end{array}$ & A & 2 \\
\hline Interno & $\begin{array}{l}\text { Servicio pos- } \\
\text { venta (man- } \\
\text { tenimientos y } \\
\text { garantías). }\end{array}$ & $\begin{array}{l}\text { Número } \\
\text { de clientes } \\
\text { atendidos en } \\
\text { posventa. }\end{array}$ & $\mathrm{F}$ & 2 \\
\hline Interno & $\begin{array}{l}\text { Alto número } \\
\text { de existencias } \\
\text { y baja rota- } \\
\text { ción de las } \\
\text { mismas. }\end{array}$ & $\begin{array}{l}\text { Rotación de } \\
\text { inventarios = } \\
\text { costo de ventas } \\
\text { / inventarios. }\end{array}$ & $D$ & 2 \\
\hline Social & $\begin{array}{l}\text { Juegos Pana- } \\
\text { mericanos } \\
2019\end{array}$ & $\begin{array}{l}\text { Número de } \\
\text { ventas reali- } \\
\text { zadas para a } \\
\text { preparación de } \\
\text { este evento. } \\
\text { Crecimiento de } \\
\text { las ventas en } \\
\text { Lima, Perú. }\end{array}$ & $\mathrm{O}$ & 2 \\
\hline Interno & $\begin{array}{l}\text { Portafolio } \\
\text { poco variado }\end{array}$ & $\begin{array}{l}\text { Número de } \\
\text { productos en el } \\
\text { portafolio. }\end{array}$ & $D$ & 2 \\
\hline
\end{tabular}

\section{Matriz de impactos cruzados}

En la matriz de impactos cruzados se confrontan las variables entre sí y se asigna un número de $\mathrm{O}$ a 3 , siendo 0 : magnitud nula, 1 : débil, 2: mediana y 3: alta magnitud del impacto. Primero se evalúa la influencia y en la siguiente situación la dependencia para dar prioridad a los factores. 
MATRIZ DE IMPACTOS CRUZADOS

\begin{tabular}{|c|c|c|c|c|c|c|c|c|c|c|c|}
\hline & V1 & $\mathbf{V} 2$ & v3 & V4 & V5 & v6 & V7 & V8 & V9 & V10 & DEPENDENCIAS \\
\hline V1 & & 1 & 1 & 2 & 0 & 2 & 1 & 0 & 0 & 0 & 7 \\
\hline V2 & 1 & & 1 & 3 & 1 & 1 & 0 & 2 & 1 & 3 & 13 \\
\hline V3 & 2 & 3 & & 0 & 1 & 2 & 3 & 2 & 3 & 2 & 18 \\
\hline V4 & 1 & 3 & 1 & & 0 & 2 & 1 & 2 & 0 & 2 & 12 \\
\hline V5 & 0 & 0 & 2 & 1 & & 0 & 2 & 1 & 3 & 1 & 10 \\
\hline V6 & 0 & 3 & 1 & 1 & 0 & & 0 & 2 & 1 & 1 & 9 \\
\hline V7 & 0 & 2 & 3 & 2 & 1 & 0 & & 1 & 1 & 0 & 10 \\
\hline V8 & 0 & 2 & 2 & 1 & 1 & 3 & 1 & & 2 & 1 & 12 \\
\hline v9 & 0 & 2 & 2 & 1 & 3 & 2 & 1 & 3 & & 3 & 17 \\
\hline V10 & 0 & 2 & 2 & 2 & 1 & 3 & 1 & 2 & 2 & & 15 \\
\hline INFLUENCIA & 4 & 18 & 14 & 13 & 8 & 15 & 10 & 15 & 13 & 13 & \\
\hline
\end{tabular}

INFLUENCIA

Figura 1. M triz de impactos cruzados

Fuente: elaboración propia (2019).

\section{Mic-Mac}

En este gráfico se encuentran cuatro cuadrantes: en el eje $Y$ se ubica la influencia y en el eje $X$ la dependencia. El primer cuadrante corresponde a las variables de riesgo o clave; en el segundo cuadrante están las variables de entrada o influyentes; en el tercer cuadrante están las variables excluidas o independientes y en el cuarto cuadrante están las variables dependientes o de salida. 


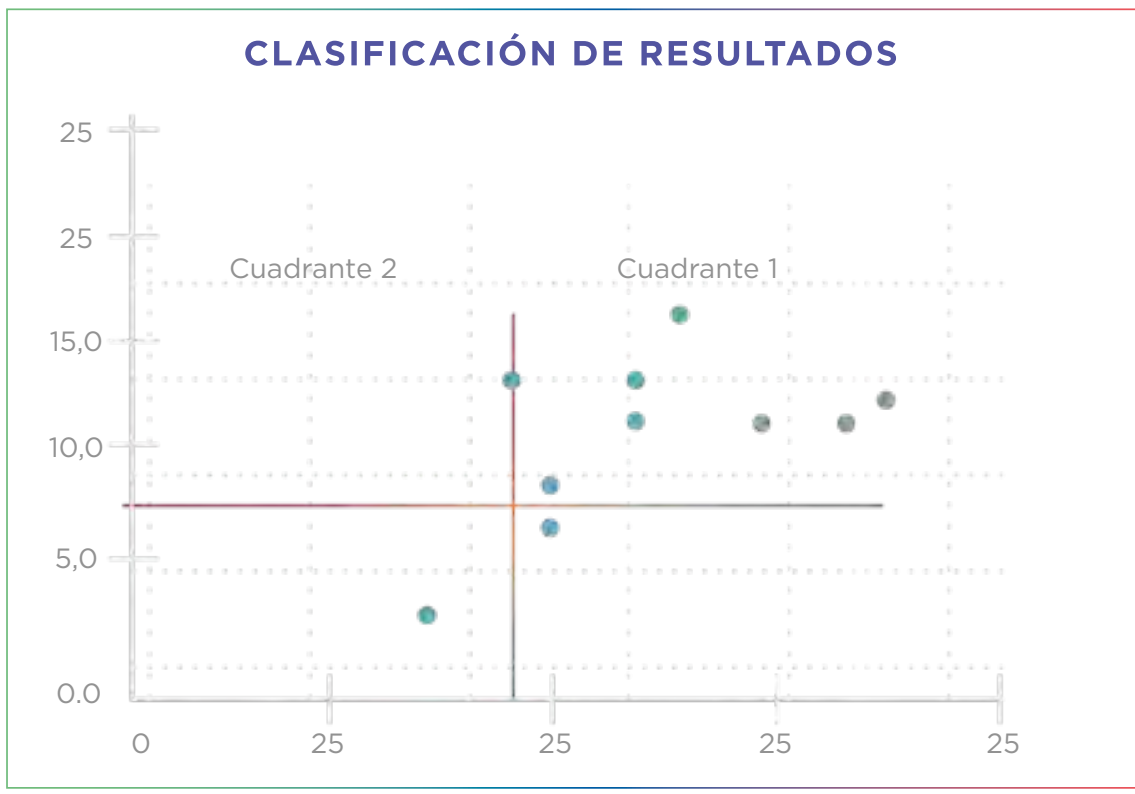

Figura 2. Mic-Mac

Fuente: elaboración propia (2019).

De acuerdo con el resultado, la mayoría de las variables se concentran en el Cuadrante 1. Esto significa que son problemas muy influyentes y altamente dependientes. Cualquier variación de ellos se verá reflejado su efecto en la zona salida o Cuadrante 4, lo que significa que se convierten en altamente dependientes, pero no son muy influyentes. 


\section{Hipótesis}

El último paso para determinar los escenarios es postular 3 hipótesis por cada variable. Cada variable debe contar con una hipótesis muy positiva, otra más realista y otra pesimista, permitiendo crear los diferentes escenarios. También se debe asignar un porcentaje a cada hipótesis teniendo en cuenta la probabilidad de cumplir con la hipótesis planteada. Esto se hace con el fin de crear diferentes escenarios en donde se puedan realizar cambios de las hipótesis de acuerdo con cada variable. Por ejemplo, el escenario más probable es el que toma cada hipótesis más probable y las une siendo este el primer escenario en el software prospectivo ya que el orden se presenta de acuerdo con la mayor probabilidad.

\section{Tabla 3. Matriz de hipótesis}

\begin{tabular}{|c|c|c|c|}
\hline $\begin{array}{l}\text { NOMBRE DE } \\
\text { LA VARIABLE }\end{array}$ & HIPÓTESIS & $\begin{array}{l}\text { RESULTADOS } \\
\text { POTENCIALES }\end{array}$ & $\begin{array}{c}\text { PROBABILIDAD } \\
\text { DEL } \\
\text { RESULTADO }\end{array}$ \\
\hline \multirow{3}{*}{$\begin{array}{l}\text { Productos } \\
\text { sustitutos pistolas } \\
\text { de pintura de } \\
\text { turbina y de } \\
\text { compresor, } \\
\text { rodillos } \\
\text { recargables). }\end{array}$} & 1 & $\begin{array}{l}\text { Ampliación del } \\
\text { portafolio en } 35 \% \text {. }\end{array}$ & 0,15 \\
\hline & 2 & $\begin{array}{l}\text { Ampliación del } \\
\text { portafolio en } 15 \% .\end{array}$ & 0,25 \\
\hline & 3 & $\begin{array}{l}\text { Ampliación del } \\
\text { portafolio en } 5 \% .\end{array}$ & 0,6 \\
\hline \multirow{3}{*}{$\begin{array}{l}\text { Calidad del } \\
\text { producto, } \\
\text { mano de obra } \\
\text { calificada. }\end{array}$} & 4 & $\begin{array}{l}\text { Obtener un certifi- } \\
\text { cado de calidad. }\end{array}$ & 0,1 \\
\hline & 5 & $\begin{array}{l}\text { Mejorar la calidad } \\
\text { del producto } \\
\text { en cuanto a las } \\
\text { materias primas } \\
\text { utilizadas. }\end{array}$ & 0,3 \\
\hline & 6 & $\begin{array}{l}\text { Mantener la } \\
\text { calidad actual. }\end{array}$ & 0,6 \\
\hline
\end{tabular}




\begin{tabular}{|c|c|c|c|}
\hline \multirow{3}{*}{$\begin{array}{c}\text { Sector en } \\
\text { crecimiento. }\end{array}$} & 7 & $\begin{array}{l}\text { Crecimiento del } \\
\text { sector mayor a } 5 \% .\end{array}$ & 0,25 \\
\hline & 8 & $\begin{array}{l}\text { Crecimiento del } \\
\text { sector en un } 3,8 \% \text {. }\end{array}$ & 0,55 \\
\hline & 9 & $\begin{array}{l}\text { Crecimiento del } \\
\text { sector inferior al 3\%. }\end{array}$ & 0,2 \\
\hline \multirow{3}{*}{ Rodillo eléctrico. } & 10 & $\begin{array}{l}\text { Vender rodillos } \\
\text { eléctricos junto con } \\
\text { los tradicionales. }\end{array}$ & 0,2 \\
\hline & 11 & $\begin{array}{l}\text { Realizar acciones } \\
\text { para promocionar } \\
\text { los rodillos actua- } \\
\text { les mostrando sus } \\
\text { beneficios. }\end{array}$ & 0,55 \\
\hline & 12 & $\begin{array}{l}\text { Mantener los rodi- } \\
\text { llos actuales y no } \\
\text { hacer nada frente } \\
\text { a la competencia } \\
\text { de los rodillos } \\
\text { eléctricos. }\end{array}$ & 0,25 \\
\hline \multirow{3}{*}{$\begin{array}{l}\text { Servicio posventa } \\
\text { (mantenimientos } \\
\text { y garantías). }\end{array}$} & 13 & $\begin{array}{l}\text { Aumentar el } \\
\text { seguimiento de } \\
\text { las ventas por lo } \\
\text { menos en } 80 \% .\end{array}$ & 0,1 \\
\hline & 14 & $\begin{array}{l}\text { Aumentar el } \\
\text { seguimiento de } \\
\text { las ventas por lo } \\
\text { menos en } 50 \% .\end{array}$ & 0,55 \\
\hline & 15 & $\begin{array}{l}\text { Mantener segui- } \\
\text { miento de ventas } \\
\text { a clientes actuales. }\end{array}$ & 0,35 \\
\hline
\end{tabular}




\begin{tabular}{|c|c|c|c|}
\hline \multirow{3}{*}{$\begin{array}{c}\text { Alto stock de } \\
\text { inventarios y baja } \\
\text { rotación de estos. }\end{array}$} & 16 & $\begin{array}{l}\text { Realizar alian- } \\
\text { zas con otros } \\
\text { distribuidores o } \\
\text { acuerdos para } \\
\text { vender en otros } \\
\text { países. }\end{array}$ & 0,3 \\
\hline & 17 & $\begin{array}{l}\text { Realizar promo- } \\
\text { ciones o reducir el } \\
\text { precio para rotar } \\
\text { los productos en } \\
\text { existencias. }\end{array}$ & 0,12 \\
\hline & 18 & $\begin{array}{l}\text { Mantener la rota- } \\
\text { ción del inventario } \\
\text { actual. }\end{array}$ & 0,58 \\
\hline \multirow{3}{*}{$\begin{array}{c}\text { Juegos } \\
\text { Panamericanos } \\
\text { Lima } 2019\end{array}$} & 19 & $\begin{array}{l}\text { Proveer más } \\
\text { del } 60 \% \text { de } \\
\text { herramientas de } \\
\text { pintura para las } \\
\text { instalaciones. }\end{array}$ & 0,15 \\
\hline & 20 & $\begin{array}{l}\text { Proveer al menos } \\
\text { el } 20 \% \text { de herra- } \\
\text { mientas de pintura } \\
\text { para las instalacio- } \\
\text { nes. }\end{array}$ & 0,6 \\
\hline & 21 & $\begin{array}{l}\text { No vender rodillos } \\
\text { para los Juegos } \\
\text { Panamericanos. }\end{array}$ & 0,25 \\
\hline
\end{tabular}




\begin{tabular}{|c|c|l|l|}
\hline \multirow{2}{*}{\begin{tabular}{|l|l|} 
Portafolio poco \\
variado
\end{tabular}} & 22 & $\begin{array}{l}\text { Implementar } \\
\text { otros productos } \\
\text { en el portafolio } \\
\text { realizando un } \\
\text { convenio con } \\
\text { otras empresas } \\
\text { aprovechando la } \\
\text { red de distribu- } \\
\text { ción. }\end{array}$ & 0,6 \\
\cline { 2 - 4 } & 23 & $\begin{array}{l}\text { Implementar } \\
\text { pocos productos } \\
\text { en el portafolio } \\
\text { como pinturas. }\end{array}$ & 0,25 \\
\cline { 3 - 5 } & \multirow{2}{*}{24} & $\begin{array}{l}\text { Mantener el } \\
\text { portafolio como } \\
\text { está actualmente. }\end{array}$ & 0,15 \\
\hline
\end{tabular}

Fuente: elaboración propia (2019).

\section{Escenarios}

De acuerdo con las fases realizadas anteriormente se obtuvieron los escenarios. Bajo el criterio del equipo de trabajo se tomaron dos de ellos: el primero y el tercero.

\section{Primer escenario}

\section{Tabla 4. Primer escenario}

\begin{tabular}{|l|l|}
\hline \multicolumn{1}{|c|}{ VARIABLES } & HIPÓTESIS \\
\hline $\begin{array}{l}\text { Productos sustitutos (pistolas de } \\
\text { pintura turbina y de compresor, } \\
\text { rodillos recargables). }\end{array}$ & Ampliación del portafolio en un 5\%. \\
\hline $\begin{array}{l}\text { Calidad del producto, mano de } \\
\text { obra calificada. }\end{array}$ & Mantener la calidad actual. \\
\hline Sector en crecimiento. & Crecimiento del sector en un $3,8 \%$. \\
\hline
\end{tabular}




\begin{tabular}{|l|l|}
\hline Rodillo eléctrico. & $\begin{array}{l}\text { Vender rodillos eléctricos junto con } \\
\text { los tradicionales. }\end{array}$ \\
\hline $\begin{array}{l}\text { Servicio posventa (mantenimientos } \\
\text { y garantías). }\end{array}$ & $\begin{array}{l}\text { Aumentar el seguimiento de las } \\
\text { ventas por lo menos en el 50\%. }\end{array}$ \\
\hline $\begin{array}{l}\text { Alto stock de inventarios y baja } \\
\text { rotación de los mismos. }\end{array}$ & $\begin{array}{l}\text { Mantener la rotación del inventario } \\
\text { actual. }\end{array}$ \\
\hline Juegos Panamericanos Lima 2019. & No vender rodillos. \\
\hline Portafolio poco variado. & $\begin{array}{l}\text { Implementar pocos productos en el } \\
\text { portafolio, tales como pinturas. }\end{array}$ \\
\hline
\end{tabular}

Fuente: elaboración propia (2019).

El primer escenario se compone de 8 variables con su respectiva hipótesis. Este escenario resulta el más probable con un $44,8 \%$ de probabilidad. En este caso, las variables se encuentran con su hipótesis más probable. De acuerdo con el software prospectivo, 3 de estas hipótesis son pesimistas, 4 son realistas y la restante es optimista. Es decir, que de acuerdo con la probabilidad asignada para el equipo de trabajo generalmente son más probables las hipótesis realistas.

Para este primer escenario se planteó la siguiente estrategia: ampliación del portafolio de productos - pinturas.

Teniendo en cuenta los factores y los valores encontrados en este escenario, se plantea una ampliación del portafolio de servicios en pinturas. Gracias a esto se puede llegar a realizar convenios (sea con empresas públicas o privadas) de un servicio más completo, ofreciéndole al cliente mayor facilidad en la búsqueda de estos productos.

\section{Plan táctico:}

1. Negociar con proveedores el nuevo producto y las diferentes variedades que se van a incluir en el portafolio.

2. Establecer políticas con proveedores.

3. Adecuar el espacio necesario para el almacenamiento de este producto. 
4. Promocionar los nuevos productos del portafolio.

5. Captación de nuevos clientes.

6. Gestionar la logística necesaria para la distribución.

7. Establecer políticas de servicio posventa.

\section{Segundo escenario}

\section{Tabla 5. Segundo escenario}

\begin{tabular}{|l|l|}
\hline \multicolumn{1}{|c|}{ VARIABLES } & \multicolumn{1}{|c|}{ HIPÓTESIS } \\
\hline $\begin{array}{l}\text { Productos sustitutos (pistolas de } \\
\text { pintura turbina y de compresor, } \\
\text { rodillos recargables). }\end{array}$ & Ampliación del portafolio en un 5\%. \\
\hline $\begin{array}{l}\text { Calidad del producto, mano de } \\
\text { obra calificada. }\end{array}$ & Mantener la calidad actual. \\
\hline Sector en crecimiento. & Crecimiento del sector mayor al 5\%. \\
\hline $\begin{array}{l}\text { Rodillo eléctrico. } \\
\text { Servicio posventa (mantenimientos } \\
\text { y garantías). }\end{array}$ & $\begin{array}{l}\text { Vender rodillos eléctricos junto con } \\
\text { los tradicionales. }\end{array}$ \\
\hline $\begin{array}{l}\text { Alto stock de inventarios y baja } \\
\text { rotación de los mismos. }\end{array}$ & $\begin{array}{l}\text { Mantener la rotación del inventario } \\
\text { actual. }\end{array}$ \\
\hline Juegos Panamericanos Lima 2019. & No vender rodillos. \\
\hline Portafolio poco variado. & $\begin{array}{l}\text { Implementar otros productos en el } \\
\text { portafolio realizando un convenio } \\
\text { con otras empresas aprovechando } \\
\text { la red de distribución. }\end{array}$ \\
\hline
\end{tabular}


En el segundo escenario los resultados no son muy distintos al primero. Ello se debe al orden en el que se eligieron los escenarios, ya que los primeros escenarios son los más probables; por lo tanto, las diferencias se encuentran en dos variables:

1. Sector en crecimiento: en este caso, en comparación con primer escenario, se cambia la hipótesis realista por la optimista debido al porcentaje de probabilidad.

2. Portafolio poco variado: la hipótesis cambió de ser realista a ser optimista.

Para el segundo escenario se planteó la siguiente estrategia:

1. Diversificación concéntrica del portafolio de servicios.

2. Implementar dentro del portafolio de servicios aquellos productos sustitutos que amenazan directamente el portafolio actual de la compañía: las pistolas de pintura de turbina y de compresor, los rodillos recargables y rodillos eléctricos.

3. La empresa debe adaptarse a los cambios tecnológicos que se presentan en la actualidad y empezar a incluir dentro de su portafolio nuevos productos que le ofrezcan al cliente mayores beneficios. La gran red de distribución de la empresa facilitará llegar a los clientes con estos nuevos productos.

\section{Plan de acción:}

1. Negociar con proveedores los nuevos productos que se van a incluir en el portafolio.

2. Establecer políticas con proveedores.

3. Adecuar el espacio necesario para el almacenamiento de estos equipos.

4. Promocionar los nuevos productos del portafolio.

5. Captación de nuevos clientes. 
6. Gestionar la logística necesaria para la distribución.

7. Establecer políticas de servicio posventa.

\section{Tercer escenario}

\section{Tabla 6. Tercer escenario}

\begin{tabular}{|l|l|}
\hline \multicolumn{1}{|c|}{ VARIABLES } & \multicolumn{1}{|c|}{ HIPÓTESIS } \\
\hline $\begin{array}{l}\text { Productos sustitutos (pistolas de } \\
\text { pintura turbina y de compresor, } \\
\text { rodillos recargables). }\end{array}$ & Ampliación del portafolio en un 5\%. \\
\hline $\begin{array}{l}\text { Calidad del producto, mano de } \\
\text { obra calificada. }\end{array}$ & Mantener la calidad actual. \\
\hline Sector en crecimiento. & Crecimiento del sector inferior al 3\%. \\
\hline Rodillo eléctrico. & $\begin{array}{l}\text { Vender rodillos eléctricos junto con } \\
\text { los tradicionales. }\end{array}$ \\
\hline $\begin{array}{l}\text { Servicio posventa (mantenimientos } \\
\text { y garantías). }\end{array}$ & $\begin{array}{l}\text { Aumentar el seguimiento de las } \\
\text { ventas por lo menos en el 50\%. }\end{array}$ \\
\hline $\begin{array}{l}\text { Alto stock de inventarios y baja } \\
\text { rotación de los mismos. }\end{array}$ & $\begin{array}{l}\text { Mantener la rotación del inventario } \\
\text { actual. }\end{array}$ \\
\hline Juegos Panamericanos Lima 2019. & No vender rodillos. \\
\hline Portafolio poco variado. & $\begin{array}{l}\text { Mantener el portafolio como está } \\
\text { actualmente. }\end{array}$ \\
\hline
\end{tabular}

Fuente: elaboración propia (2019)

Para el tercer escenario ocurre algo similar al segundo, dado que son las mismas variables las que cambian.

1. Crecimiento en el sector: su hipótesis cambia de ser realista a ser pesimista.

2. Portafolio poco variado: cambian las hipótesis a las menos probables de las 3 existentes, esto debido a que en este escenario se eligen las hipótesis menos probables. 
Para este tercer escenario se planteó la siguiente estrategia:

1. Fortalecimiento de procesos internos como el seguimiento en ventas y la rotación del inventario. Estas dos actividades presentan algunas debilidades en la organización y su fortalecimiento conlleva a una mejoría tanto para el cliente en el servicio posventa, como para la disminución de costos que incurren en el almacenamiento del inventario.

\section{Plan táctico}

Respecto al servicio de posventa:

1. Evaluar la política actual de servicio al cliente y establecer si es necesario modificar o reestructurarla.

2. Evaluar si los actuales canales de comunicación son efectivos y si es necesario implementar nuevos o cerrar aquellos que sean obsoletos.

Respecto a la rotación de inventarios:

3. Evaluar los costos en los que se está incurriendo con alto stock de inventario.

4. Revisar cuál es o cuáles son los productos que menos rotan y reevaluar su plan de producción.

5. Crear una política de promociones para aumentar la rotación de aquellos productos que se encuentran estancados en inventarios, siempre y cuando sea rentable hacerlo. 


\section{CONCLUSIONES}

El portafolio poco variado es una de las principales desventajas de Rodillos Khan en comparación con su competencia. La tecnología ha permitido tecnificar diferentes procesos y la comercialización de rodillos para pintar no es la excepción. Una de las grandes amenazas para esta empresa son los productos sustitutos que ofrecen mayores beneficios al cliente, y es evidente que Rodillos Khan se ha quedado atrás en estas nuevas tecnologías.

Gracias al software de prospectiva se logró establecer varios escenarios posibles. Escenarios en los cuales se enfrentan las desventajas y amenazas con las que cuenta actualmente la empresa. Dicho software no arroja una única solución a los problemas, por el contrario, ofrece una variedad de soluciones que se pueden acoplar a las necesidades de la empresa y permitir su estabilidad en el mercado.

Cada uno de los escenarios cuenta con un nivel de probabilidad de cumplimiento. En este caso, se eligieron los tres primeros con mayor probabilidad. Sin embargo, cada variable cuenta con diferentes cambios en cada una de sus hipótesis; lo que nos permitió plantear una estrategia diferente para cada uno de los casos.

En el primer escenario se puede inferir que, dadas sus hipótesis, la estrategia a implementar no será de gran impacto. Lo anterior dado que lo que se propone es agregar un producto complementario o adicional a los productos con los cuales cuenta actualmente la empresa.

En el segundo escenario la estrategia es de un impacto mayor, siendo así la más agresiva de las tres estrategias. Esta propone atacar la más fuerte de sus amenazas incluyendo dentro de su portafolio aquellos productos sustitutos que amenazan directamente el portafolio actual.

En el tercer escenario la estrategia va enfocada a nivel interno, al fortalecimiento y correcta ejecución de sus funciones internas. En el caso de Rodillos Khan, se verían involucradas los procesos de servicio posventa y rotación de inventarios. 


\section{RECOMENDACIONES}

Estudiar la posibilidad de exportar directamente a Bolivia, pues este país pertenece a la Comunidad Andina al igual que Colombia, Perú y Ecuador. Gracias a esta alianza los gravámenes arancelarios son menores. Además, el sector de construcciones en este país ha venido presentando un crecimiento, por lo que exportar a dicho país es una gran oportunidad para Rodillos Khan.

Crear un área de exportaciones en la cual se realice un seguimiento a cada uno de los envíos hacia los diferentes países. Esto permitirá evaluar y darles control a estas alianzas.

A diario se puede evidenciar el avance tecnológico que se vive en la actualidad. Gracias a ello, se han reducido los tiempos invertidos en una gran cantidad de procesos y eso es lo que busca el consumidor actualmente. Por ende, Rodillos Khan debe incluir dentro de su portafolio productos más tecnificados que le permitan llegar a nuevos clientes.

La empresa debe cambiar la infraestructura física de una de las fábricas pues actualmente se encuentra en mal estado y podría ocasionar accidentes, una reparación del lugar le ofrecerá al colaborador mayor seguridad en su puesto de trabajo. 


\section{BIBLIOGRAFÍA}

Becerra, L. L. (28 de febrero de 2019). Producto Interno Bruto de Colombia creció 2,7\% en 2018 según informó el Dane. La República. Recuperado de https://www.larepublica.co/economia/productointerno-bruto-de-colombia-crecio-27-en-2018-segun-informo-eldane-2834007

Carreño, L., Mamián, K., Díaz, C. y Moreno, C. (2018). Diagnóstico Rodillos Khan. Bogotá D.C: Politécnico Grancolombiano.

Flórez, C. R. (8 de Enero de 2019). Sector construcción repuntará en 2019 con más inversión y generación de empleo. La República. Recuperado de https://www.larepublica.co/empresas/construccion-repuntara-en-2019-en-cuanto-a-inversion-y-generacion-deempleo-2812447

Gabiña, J. (1995). El futuro revisitado. Barcelona: Marcombo.

Godet, M. (2007). Prospectiva estratégica: Problemas y métodos. París: Prospektiker.

Lira, G. (20 de marzo de 2018). Cerca de U\$500 millones para los Panamericanos 2019. CLA Construcción Latinoamericana. Recuperado de https://www.construccionlatinoamericana.com/news/Cerca-de-US-500-millones-para-los-Panamericanos-2019/4132140.article

Leroy Merlin. (2019). Pistolas de pintura. España. Recuperado de https://www.leroymerlin.es/buscador?q=pistolas\%20de\%20pintura

Martín-Carrillo, S. (16 de diciembre de 2018). La economía boliviana en 2019. CELAG. Recuperado de https://www.celag.org/ la-economia-boliviana-2019/

Ministerio de Economía y Finanzas Públicas. (2017). La economía de Bolivia crecerá en 4,7\% en 2018. ECONoticias, 3(1), 1-16. Recuperado de https://medios.economiayfinanzas.gob.bo/MH/documentos/2018/mefp/Periodico_1_-_2018.pdf 
Ministerio de Industria, Comercio y Turismo. (2019). Informe de la industria manufacturera a enero de 2019. Recuperado de https://www.mincit.gov.co/getattachment/433a0476-f1ef-4a27-8af5-b2783c341509/Enero.aspx

Pulgar, L. H. y Ramos, F. R. (2011). Metodología para implantar la estrategia (2da. Ed.). Lima: Universidad Peruana de Ciencias Aplicadas S.A.C.

Redacción EC. (21 de mayo de 2018). El sector construcción creció en $5,1 \%$ en el primer trimestre. El Comercio. Recuperado de https://elcomercio.pe/economia/peru/sector-construccion-crecio-5-1-primer-trimestre-noticia-521581-noticia/?ref=ecr

Redacción Portafolio. (23 de Agosto de 2018). Exportación de materiales para la construcción crecerán $5 \%$ en 4 años. Portafolio. Recuperado de https://www.portafolio.co/economia/colombia-liderara-exportaciones-de-materiales-de-construccion-en-la-region-520358

Redacción Portafolio. (8 de enero de 2019). Alzas en energía eléctrica, las reinas de la inflación en 2018. Portafolio. Recuperado de https://www.portafolio.co/economia/alzas-en-energia-electricalas-reinas-de-la-inflacion-en-2018-525032

Rodríguez-Castillo, E. (2010). Aplicación de la planeación estratégica en el centro de investigaciones del níquel. Ingeniería Industrial, 21(3), 1-6. 
